\title{
Elevated DNA methylation in malignant tumors of the sinonasal tract and its association with patient survival
}

\author{
Marcela Chmelarova a , Jan Laco ${ }^{b}$, Helena Kovarikovaa ${ }^{a}$, Ivana Baranova a , Pavel Dundrc, Kristyna Nemejcovac, \\ Jaroslav Michalek ${ }^{\mathrm{d}}$, Milan Vosmike, Vladimir Palicka ${ }^{\mathrm{a}}$
}

Background. Epigenetic modifications have been recognized as an important mechanism underlying carcinoma progression. DNA methylation plays an important role in cancer biology and represents potentially heritable changes in gene expression that do not involve DNA sequence. The aim of this study was to investigate promoter methylation of selected genes in sinonasal carcinoma by comparison with noncancerous sinonasal tissue.

Methods. To search for epigenetic events (methylation in 25 tumor suppressor genes) we used MS-MLPA (Methylationspecific multiplex ligation-dependent probe amplification) to compare methylation status of 59 formalin fixed, paraffin embedded tissue samples of sinonasal carcinomas with 18 control samples. The most important changes in methylation were confirmed using MSP (Methylation specific PCR). Detected alterations in methylation were compared with clinicopathological characteristics.

Results. Using a 20\% cut-off for methylation (MS-MLPA), we found significantly higher methylation in GATA5 $(P=0.0005)$, THSB1 $(P=0.0002)$ and PAX5 $(P=0.03)$ genes in the sinonasal cancer group compared to the control group. Methylation in five or more genes was associated with impaired overall survival $(P=0.017)$.

Conclusion. These findings provide evidence that alterations in methylation profile may be one of the major mechanisms in sinonasal carcinogenesis. In addition, changes in methylation could potentially be used as prognostic factors of sinonasal carcinoma and may have implications for future individualized therapy based on epigenetic changes.

Key words: biomarkers, DNA methylation, sinonasal carcinoma, epigenetics

Received: November 27, 2017; Accepted: March 21, 2018; Available online: March 27, 2018

https://doi.org/10.5507/bp.2018.012

aInstitute for Clinical Biochemistry and Diagnostics, Charles University, Faculty of Medicine in Hradec Kralove and University Hospital Hradec Kralove, Czech Republic

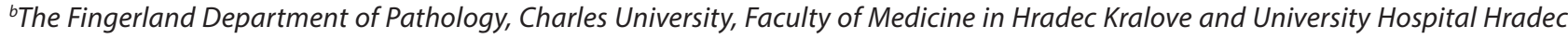
Kralove, Czech Republic

'Department of Pathology, First Faculty of Medicine, Charles University in Prague and General University Hospital in Prague, Czech Republic ${ }^{d}$ Department of Clinical and Molecular Pathology, Faculty of Medicine and Dentistry, Palacky University Olomouc and University Hospital Olomouc, Czech Republic

${ }^{e}$ Department of Oncology and Radiotherapy, Charles University, Faculty of Medicine in Hradec Kralove and University Hospital Hradec Kralove, Czech Republic

Corresponding author: Marcela Chmelarova, e-mail: ChmelarovaMarcela@seznam.cz

\section{INTRODUCTION}

Malignant tumors of the sinonasal tract are rare tumors of the head and neck area that account for approximately $3 \%$ to $5 \%$ of all upper respiratory tract malignancies ${ }^{1}$. In 2014, 49 new cases in men and 39 in women were diagnosed in the Czech Republic, giving the incidence rate $0.84 / 100,000\left(\right.$ ref. $\left.^{2}\right)$. These figures probably reflect the general status in Europe and worldwide ${ }^{3-4}$. The average age at which patients are diagnosed with sinonasal carcinomas is between 50-60 years. However, there are some risk factors including exposure to wood and leather dust, tabaco smoke exposure, contact with chemical substances such as formaldehyde, chrome pigment, nickel and asbestos and HPV infection ${ }^{5}$. Diagnosis and treatment of these tumors pose several problems due to their very low incidence, histological diversity and production of non-specific symptoms in the early stages that can closely mimic inflammatory conditions. Their prognosis largely depends on histology, location and staging ${ }^{6}$.

Sinonasal carcinoma has been considered as a disease driven by progressive genetic alterations, such as mutations involving oncogenes or tumor suppressor genes, as well as chromosomal abnormalities ${ }^{5}$. In addition, approximately $20-30 \%$ of these tumors harbor transcriptionally active high risk human papillomavirus infection ${ }^{7}$. More recently, it has been demonstrated that sinonasal cancer is also driven by epigenetic alterations ${ }^{8.9}$. Currently, DNA methylation is one of the most broadly studied and wellcharacterized epigenetic modifications. Our knowledge about it is dating back to 1969, when Griffith and Mahler suggested that DNA methylation may be important in long term memory function ${ }^{10}$. DNA methylation is mediated by DNA methyltransferases, which catalyze the covalent addition of a methyl group to the 5-carbon of the cytosine in $\mathrm{CpG}$ context dispersed throughout the 
genome or in DNA repetitive regions. Promoter DNA methylation at $\mathrm{CpG}$ sites represses gene expression by impeding access to transcription factors and inhibiting RNA polymerase II (ref. ${ }^{11}$ ). In cancer, aberrant DNA methylation is typically observed in the promoter/exon 1 regions of various tumor suppressor genes ${ }^{12}$, transcription factors ${ }^{13}$, DNA repair genes and cell cycle regulators leading to their transcriptional silence ${ }^{14}$. However, various tumor types have different patterns of hypermethylated genes.

As sinonasal carcinomas are group of aggressive tumors with poor prognosis, it is very important to know their molecular parameters to be able to establish diagnostic strategies and individualized therapies. Sinonasal malignancies are rare, which makes it difficult to conduct extensive methylation studies. In the present study we were able to gather, thanks to cooperation of three university hospitals in Czech Republic, a huge and unique group of sinonasal cancer patients. The aim of this study was to investigate promoter methylation of specific genes in sinonasal carcinoma by comparison with nonmalignant sinonasal tissue. We are fully aware of the fact that the absence of mRNA expression analysis may be the main weakness of this study. However, we are unable to provide relevant information about gene expression of selected genes, because we were limited to work only with FFPE tissue samples, which are generally not suitable for RNA isolation and subsequent gene expression analysis due to high fragmentation of RNA.

\section{MATERIALS AND METHODS}

Formalin-fixed, paraffin-embedded tissue samples of sinonasal carcinomas and noncancerous sinonasal tissue were obtained from 77 patients: 59 patients with sinonasal cancer, and 18 patients with noncancerous sinonasal tissue. Only tumors primarily originating from the nasal cavity, maxillary sinuses and ethmoid complex were included. No tumors were found in the frontal or sphenoid sinuses. The samples of noncancerous tissue ( 9 mucosal specimens from the nasal cavity and 9 from the maxillary sinus) were obtained from patients treated for chronic rhinitis and sinusitis. The paraffin blocks were retrieved from the archives of the Fingerland Department of Pathology, University Hospital Hradec Kralove; the Department of Pathology, General University Hospital, Prague, Czech Republic; and the Department of Pathology, University Hospital Olomouc, Czech Republic. All slides were reviewed by experienced head and neck pathologist (J.L.) and the carcinomas were classified according to the current WHO classification ${ }^{15}$. The study was approved by the Ethics Committee of University Hospital Hradec Kralove. The need for informed consent was waived by the review board in view of the retrospective nature of the study and long archival period of the formalin-fixed, paraffin-embedded tissue samples involved.

For every patient were recorded data such as gender, age at the time of diagnosis, smoking history (non-smoker vs. ex-smoker vs. current smoker), occupation (risky vs. non-risky), and tumor localization, including the nasal cavity, maxillary sinus, and ethmoid complex, laterality and pathological TNM. During the follow-up period local recurrence, regional recurrence, distant recurrence, death, and tumor-related death staging were recorded. When radical surgery was not performed, clinical TNM staging was used instead. Treatment modalities were radical surgery, radiotherapy, and chemotherapy in various combinations.

The tumor types included squamous cell carcinoma (SCC), lymphoepithelial carcinoma (LEC), sinonasal undifferentiated carcinoma (SNUC), adenocarcinoma, and neuroendocrine carcinoma. Vascular invasion, perineural spread, status of resection margins (in the case of radical surgery), and microscopic findings in the surrounding mucosa were also noted.

DNA for methylation analysis was extracted from formalin-fixed, paraffin-embedded tissue samples using a Qiagen DNA extraction kit (Hilden, Germany).

\section{Methylation-specific multiplex ligation-dependent probe amplification (MS-MLPA)}

All tumor and control noncancerous samples were tested by the MS-MLPA probe set ME002 (MRC-Holland, Amsterdam, The Netherlands), which can simultaneously check for aberrant methylation in 25 tumor suppressor genes (BRCA1, BRCA2, ATM, TP53, PTEN, MGMTa, PAX5, CDH13, TP73, WT1, VHL, GSTP1, CHFR, ESR1, RB1a, MSH6, MGMTb, THBS1, CADM1, STK1, PYCARD, PAX6, $C D K N 2 A$, GATA5, RARB, CD44, RB1b). Probe sequences, gene loci and chromosome locations can be found at http://www.mlpa.com. The experimental procedure was carried out according to the manufacturer's instructions, with minor modifications as previously described ${ }^{8}$.

\section{Methylation-specific polymerase chain reaction (MSP)}

The most important genes showing highly significant methylation $(P<0.001)$ in MS-MLPA $(G A T A 4$ and $T H S B 1$ ) were further tested by means of MSP for confirmation. MSP requires bisulfite treatment of genomic DNA, which is used for conversion of all unmethylated cytosines to uracils, leaving methylated cytosines unaffected. A total of $500 \mathrm{ng}$ of genomic DNA was treated with bisulfite using the EZ DNA Methylation-Gold ${ }^{\mathrm{TM}}$ Kit according to the manufacturer's protocol (Zymo Research Corporation Irvine, CA, USA). MSP was performed on the Rotor-Gene Q (Qiagen, Hilden, Germany) in two types of reaction mixture within one run, for amplifying methylated and unmethylated DNA, respectively. Primers were designed using MethPrimer with consideration of the MS-MLPA probe locations and the FFPE DNA fragmentation. Primer sequences with amplicon lengths are listed in Table 1. MSP reaction mixture contained 10x PCR Buffer, $\mathrm{MgCl}_{2}$ (25 mM), dNTPs solution Takara (2.5 $\mathrm{mM})$, primers $(10 \mu \mathrm{M})$, Platinum ${ }^{\circledR}$ Taq DNA Polymerase (Invitrogen by Thermo Fisher Scientific, Foster City, CA, USA), SYTO ${ }^{\circledR} 9$ Dye $(0.05 \mathrm{mM})$, bisulfite converted DNA and water. Each run included a bisulfiteconverted universal methylated and unmethylated DNA (Qiagen, Hilden, Germany) and a no template control. Fluorescence data were analyzed using Rotor Gene Q 
Table 1. MSP primers and amplicon information.

\begin{tabular}{llcc}
\hline Amplicon name & Primer sequence 5‘3‘ & Amplicon size (bp) & CpGs/Primer \\
\hline GATA5-M & Fw: TTAGCGTTGGGGTTTCGGTC & 92 & Fw: 3 \\
& Rv: TAACCGCCCCGTATCGTACG & & Rv: 4 \\
GATA5-U & Fw: GGTTAGTGTTGGGGTTTTGGTTGT & 95 & Fw: 3 \\
& Rv: CTAACCACCCCATATCATACATC & & Rv: 4 \\
THBS1-M & Fw: AGCGTTTTTTTAAAAGCGCGC & 109 & Fw: 4 \\
& Rv: TCCGAAATAAAAATTACTCCTAAAAAACGA & & Rv: 2 \\
THBS1-U & Fw: GAGTGTTTTTTAAAAGTGTGTGG & 110 & Fw: 4 \\
& Rv: TCCAAAATAAAAATTACTCCTAAAAAACAA & & Rv: 2 \\
\hline
\end{tabular}

M - methylated, U - unmethylated

software. Amplicon was considered as methylated when there was amplification in reaction mixture with methylated primers or both types of reaction mixture. When there was amplification only in mixture with primer pair for unmethylated DNA, the amplicon was considered as unmethylated.

\section{Statistical analysis}

Basic descriptive statistics were adopted for the analysis: median, mean, and 95\% confidence interval for continuous data, and absolute and relative frequencies for categorical data. The relationship between gene methylation and other independent factors was analyzed using the chi-square test, Fisher's exact test, or Logistic regression analysis. Kaplan-Maier and Logrank tests were used for survival analysis; Cox regression analysis was used to determine the influence of gene methylation upon survival. We considered $P<0.05$ to be statistically significant. All statistical analyses were performed using STATISTICA $\mathrm{Cz}$ (data analysis software system) version 12 (StatSoft, Inc., Tulsa, OK, USA).

\section{RESULTS}

Using a 20\% cut-off for methylation (MS-MLPA), we observed significantly higher methylation in GATA5 $(P=0.0005)$, THBS1 $(P=0.0002)$ and $P A X 5(P=0.03)$ genes in the sinonasal cancer group compared to the control noncancerous group. For genes BRCA1, BRCA2, ATM, $V H L$, and $R B 1 a$ the methylation rate did not exceed the $20 \%$ threshold; the other genes also showed relevant differences in methylation between samples with sinonasal carcinoma and control samples (see Fig. 1). Using MSP, we confirmed significantly higher methylation of GATA5 gene in samples of sinonasal carcinoma in comparison with noncancerous sinonasal tissue $(P=0.03)$. Using MSP we weren't able to confirm the presence of methylation of THSB 1 gene in cancer samples, but it could be the result of the assay strategy ( $6 \mathrm{CpGs}$ in primers, compared to 2 $\mathrm{CpG}$ restriction sites in MS-MLPA probe).

\section{Correlation with clinicopathological features}

Clinicopathological data are listed below. Due to a few

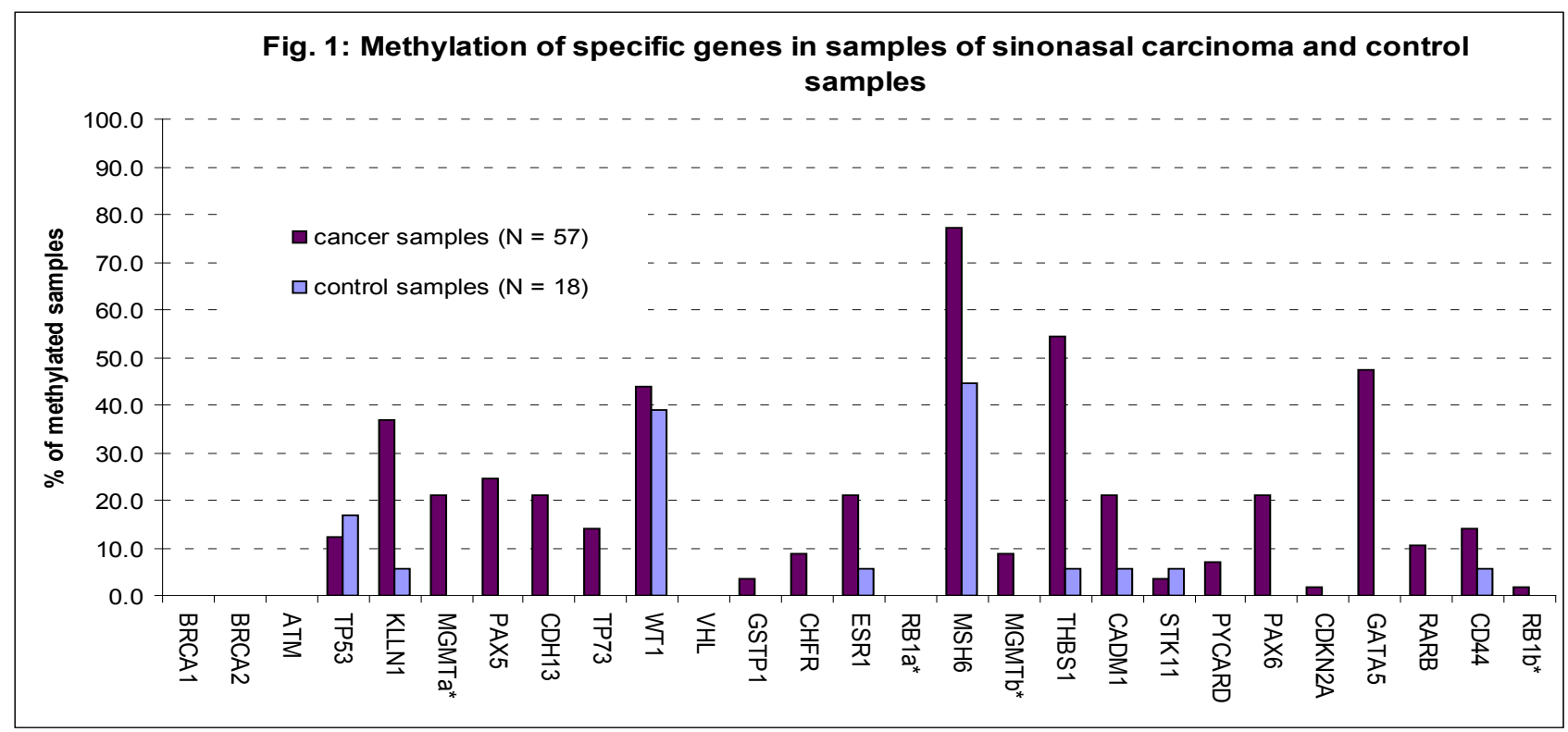

Fig. 1. Methylation of specific genes in samples of sinonasal carcinoma and control samples.

Comparison of methylation frequencies (cut-off value 20\%) of the 25 analyzed genes in sinonasal cancer and control samples. * Two CpG loci (a and b) were analyzed 


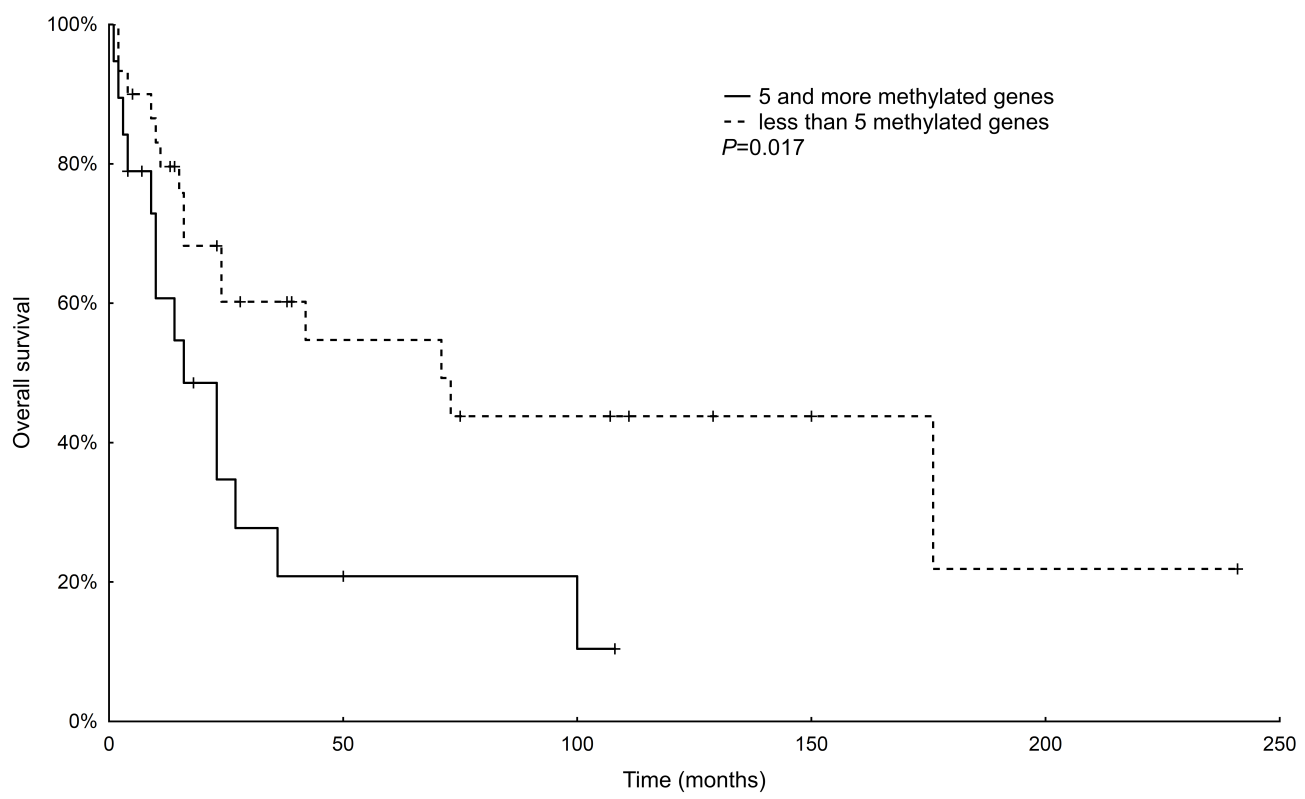

Fig. 2. Kaplan-Meier survival analysis.

Overall survival of patients according to the presence of methylation. Vertical hatch marks show censored data.

missing clinical data sums in the entire study sample or partial sums do not always add up to the total number of patients. The median age of patients at the time of diagnosis was 62 years (range 23-82 years) in the carcinoma group and 56.5 years (range 24-74 years) in the control group. The carcinoma group consisted of 39 males and 20 females and the control group 9 males and 9 females.

From those in the carcinoma group with a known history, 25 patients were non-smokers, 9 former smokers, and 17 were current smokers. In only 5/51 patients, occupational exposure to wood dust or other air pollutants/ irritants was recorded.

As regards the whole study sample, most of the tumors arose in the nasal cavity, but SCCs were slightly more common in the maxillary sinus. The majority of the patients were diagnosed with advanced tumors and three patients had lung metastases (cM1) at the time of diagnosis.

Microscopic typing of the tumors resulted in 43 SCCs, 11 adenocarcinomas, 2 neuroendocrine carcinomas, 2 SNUCs, and 1 LEC. Vascular invasion was found in 7/59 tumors and perineural spread in $3 / 59$.

The follow-up period ranged from 1 to 241 months (median 23 months). Local recurrence was found in 23/50 tumors, 5/50 recurred regionally, and 3/59 patients developed distant metastases in the lungs. During the follow-up period, 29/49 patients died, of whom 14/49 due to the tumor.

The methylation results from the carcinoma specimens were compared with clinicopathological characteristics mentioned above (Table 2). Presence of methylation in five or more genes was connected with worse overall survival $(P=0.017)$, see Fig. 2. No correlation was found between DNA methylation and tumor type, stage, grade and smoking history.

\section{DISCUSSION}

A wide array of techniques is currently available to measure DNA methylation level. In our study we used MS-MLPA a semi-quantitative method for methylation profiling. MS-MLPA allows simultaneous assessment of aberrant promoter methylation of large sets of genes and requires only small quantities of short DNA fragments, making it very suitable for analysis of DNA isolated from FFPE tissue samples ${ }^{16}$. Using DNA methylation profiling as a biomarker has a number of advantages including DNA stability, relatively low cost of testing and restriction to limited specific regions of DNA. Moreover DNA methylation analysis can be performed from small biopsy samples obtained during the routine diagnostic work-up of patients. In our study we employed the idea of a candidate - gene approach for searching the potential prognostic biomarkers in sinonasal carcinoma. Recently, a successful example of methylation-based prognostic and predictive cancer biomarker is hypermethylation of $M G M T$ promoter region, which is routinely analyzed and predicts response to temozolomide in glioblastoma patients and their clinical outcome ${ }^{17}$. Next promising biomarker is methylation of $X R C C 2$ gene predicting the occurrence of late toxicity in radiotherapy-treated cervical cancer patients ${ }^{18}$. In head and neck cancer area methylation in PITX3, SHISA3 and $F O X F 2$ in squamous cell types of carcinomas also represent promising prognostic biomarkers ${ }^{19-21}$.

In our study we observed significantly higher methylation in three genes: GATA5, THBS1 and PAX5. GATA5 methylation has been already found in hepatocellular carcinoma $^{22}$, colorectal cancer ${ }^{23}$, glioblastomas ${ }^{24}$ and ovarian cancer $^{25}$. THBS1 methylation has been demonstrated in laryngeal squamous cell carcinoma ${ }^{26}$, glioblastomas ${ }^{24}$ and ovarian cancer ${ }^{25}$ and $P A X 5$ methylation has been shown in 
Table 2. Clinicopathological characteristics and methylation.

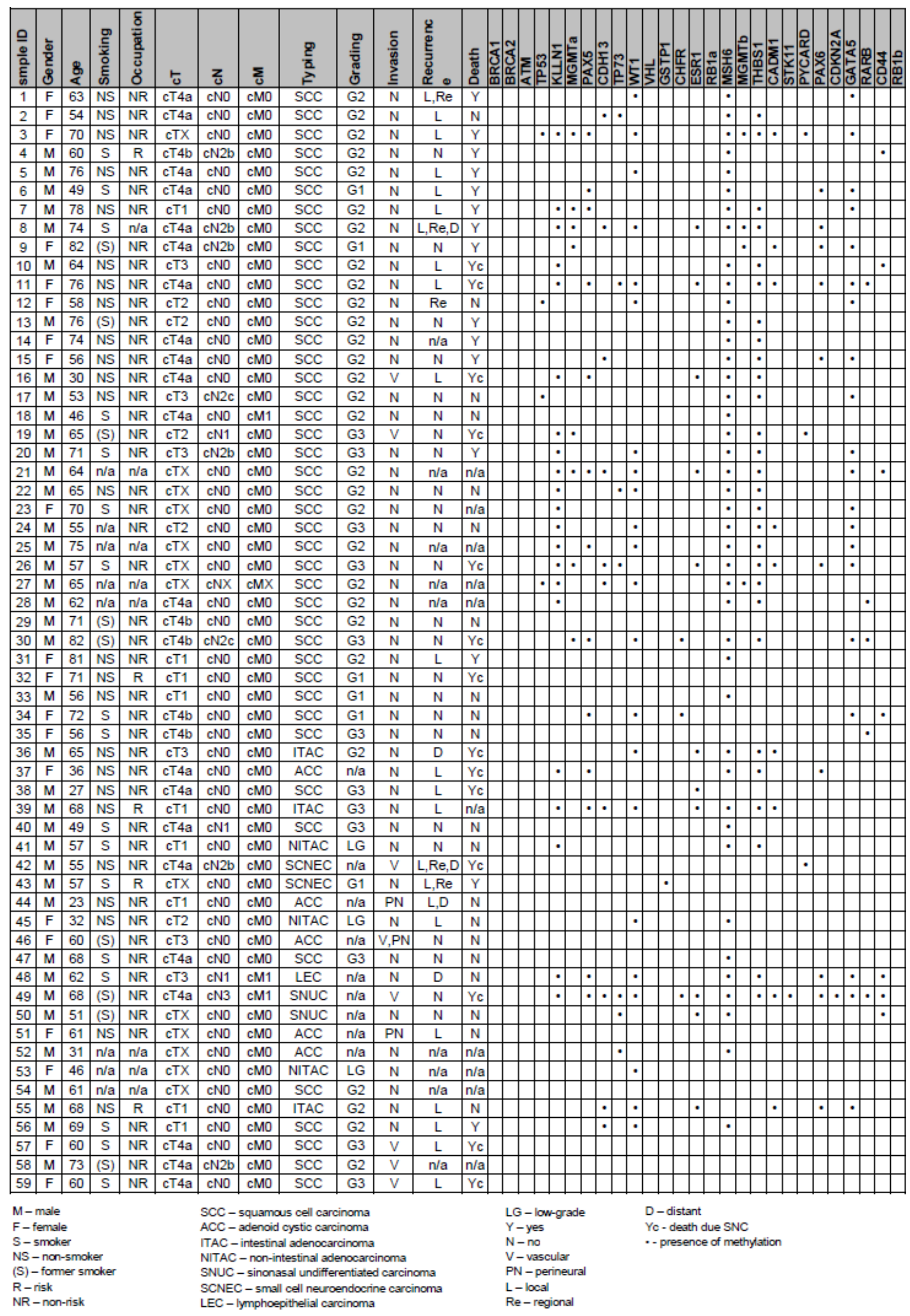


hepatocellular carcinoma ${ }^{27}$ and non-small cell lung can$\operatorname{cer}^{28}$. These findings supported the theory that methylation changes in these genes could lead to carcinogenesis also in sinonasal carcinoma.

Our experiments never showed methylation in genes BRCA1, BRCA2, ATM, VHL, and RBla, suggesting that methylation of selected $\mathrm{CpG}$ loci of these tumor suppressor genes may not play an important role in carcinogenesis of the sinonasal area. On the other hand, other genes (see Fig. 1) did show promoter methylation to a varying extent above the $20 \%$ threshold. Methylation of TP53, WT1 and MSH6 was detected in more than $10 \%$ of control samples. Presence of this methylation could be associated with nature of the control samples. All control samples were obtained from patients with inflammation in sinonasal area. At sites of chronic inflammation, epithelial cells are exposed to high levels of reactive oxygen species and undergo cancer-associated DNA methylation changes, suggesting that inflammation may initiate epigenetic alterations ${ }^{29}$. These findings correlate with research Wang et al. (2014), where methylation of TIMP3, GSTP-1 and 14-3-3 $\sigma$ was found in patients with chronic inflammation and in cancer patients ${ }^{30}$.

Methylation changes may be connected with a more aggressive phenotype and thus be associated with unfavorable clinical outcome. We demonstrated correlation between presence of methylation in five or more genes and shorter overall survival time. These findings show that accumulation of changes in methylation during sinonasal cancer development could be associated with patient's prognosis and so has a potential to serve as a biomarker in the future.

\section{CONCLUSION}

Our observations provide evidence that changes in methylation of these genes may be one of the major mechanisms in sinonasal carcinogenesis. In addition, changes in methylation could potentially be used as prognostic markers of sinonasal cancer and may have implications for future individualized therapy based on epigenetic changes. Future work with a larger number and in various ethnic groups is warranted to confirm that methylation in selected genes is a reliable prognostic biomarker for SNC patients.

Acknowledgement: The authors are grateful to Veronika Hruzova for excellent technical assistance.

The study was supported by conceptual development of research organization MH CZ-DRO (FNHK) 00179906 (Ministry of Health, Czech Republic), by the project BBMRI_CZ LM2015089 (Ministry of Education, Youth and Sports; Czech Republic), and by Research Development Program PROGRES Q40/11 (Charles University).

Author contributions: MC: study design; PD, KN, JM, MV: patient selection; JL: reviewed all pathologic and control samples. IB, HK, MC: experiments performing;
MC, IB, HK: data analysis; MC, HK: manuscript writing; VP, JL: manuscript revision; All authors: final approval. Conflict of interest statement: None declared.

\section{REFERENCES}

1. Haerle SK, Gullane PJ, Witterick IJ, Zweifel C, Gentili F. Sinonasal carcinomas: epidemiology, pathology, and management. Neurosurg Clin N Am 2013;24(1):39-49.

2. Epidemiology of malignant tumors in the Czech Republic. Available from www.svod.cz

3. Turner JH, Reh DD. Incidence and survival in patients with sinonasal cancer: a historical analysis of population-based data. Head Neck 2012;34(6):877-85.

4. Syrjänen K, Syrjänen S. Detection of human papillomavirus in sinonasal carcinoma: systematic review and meta-analysis. Hum Pathol 2013;44(6):983-91.

5. Llorente JL, López F, Suárez C,Hermsen MA. Sinonasal carcinoma: clinical, pathological, genetic and therapeutic advances. Nat Rev Clin Oncol 2014;11(8):460-72.

6. López F, Llorente JL, Costales M, García-Inclán C, Pérez-Escuredo J, Alvarez-Marcos C, Hermsen M, Suárez C. Molecular characterisation of sinonasal carcinomas and their clinical implications. Acta Otorrinolaringol Esp 2013;64(4):289-96.

7. Laco J, Sieglová $K$, Vošmiková $H$, Dundr $P$, Němejcová $K$, Michálek J, Čelakovský P, Chrobok V, Mottl R, Mottlová A, Tuček L, Slezák R, Chmelařová $M$, Sirák I, Vošmik $M$, Ryška $A$. The presence of high-risk human papillomavirus (HPV) E6/E7 mRNA transcripts in a subset of sinonasal carcinomas is evidence of involvement of HPV in its etiopathogenesis. Virchows Arch 2015;467(4):405-15.

8. Chmelarova M, Sirak I, Mzik M, Sieglova K, Vosmikova H, Dundr P, Němejcová K, Michálek J, Vošmik M, Palička V, Laco J. Importance of tumor suppressor genes methylation in sinonasal carcinomas. Folia Biol (Praha) 2016;62(3):110-9.

9. Laco J, Chmelařová $M$, Vošmiková H, Sieglová K, Bubancová I, Dundr P, Němejcová K, Michálek J, Čelakovský P, Mottl R, Sirák I, Vošmik M, Ryška A. SMARCB1/INI1-deficient sinonasal carcinoma shows methylation of RASSF1 gene: A clinicopathological, immunohistochemical and molecular genetic study of a recently described entity. Pathol Res Pract 2017;213(2):133-42.

10. Griffith JS, Mahler HR. DNA ticketing theory of memory. Nature 1969;223(5206):580-2.

11. Curradi M, Izzo A, Badaracco G, Landsberger N. Molecular mechanisms of gene silencing mediated by DNA methylation. Mol Cell Biol 2002;22(9):3157-73.

12. Chmelarova M, Krepinska E, Spacek J, Laco J, Mzik M, Palicka V. Promoter methylation of GATA4, WIF1, NTRK1 and other selected tumor suppressor genes in ovarian cancer. Folia Biol (Praha) 2013;59(2):87-92.

13. Bubancova I, Kovarikova H, Laco J, Ruszova E, Dvorak O, Palicka V, Chmelarova M. Next-generation sequencing approach in methylation analysis of HNF1B and GATA4 genes: searching for biomarkers in ovarian cancer. Int J Mol Sci 2017;18(2):pii:E474.

14. Rao-Bindal K, Kleinerman ES. Epigenetic regulation of apoptosis and cell cycle in osteosarcoma. Sarcoma 2011;679457.

15. Barnes L, Eveson JW, Reichart P and Sidransky D, editors. Tumours of the Nasal Cavity and Paranasal Sinuses. Lyon: IARC Press; 2005.

16. Nygren AO, Ameziane N, Duarte HM, Vijzelaar RN, Waisfisz Q, Hess CJ, Schouten JP, Errami A. Methylation-specific MLPA (MS-MLPA): simultaneous detection of CPG methylation and copy number changes of up to 40 sequences. Nucleic Acids Res 2005;33(14):e128.

17. laccarino C, Orlandi E, Ruggeri F, Nicoli D, Torricelli F, Maggi M, Cerasti D, Pisanello A, Pedrazzi G, Froio E, Crafa P, D'Abbiero N, Michiara M, Ghadirpour R, Servadei F. Prognostic value of MGMT promoter status in non-resectable glioblastoma after adjuvant therapy. Clin Neurol Neurosurg 2015;132:1-8.

18. Paulíková S, Chmelařová $M$, Petera J, Palička V, Paulík A. Hypermethylation of RAD51L3 and XRCC2 genes to predict late toxicity in chemoradiotherapy-treated cervical cancer patients. Folia Biol (Praha) 2013;59(6):240-5.

19. Sailer V, Holmes EE, Gevensleben H, Goltz D, Dröge F, Franzen A, Dietrich J, Kristiansen G, Bootz F, Schröck A, Dietrich D. PITX3 DNA 
methylation is an independent predictor of overall survival in patients with head and neck squamous cell carcinoma. Clin Epigenetics 2017;9:12. doi: 10.1186/s13148-017-0317-7. eCollection

20. Shen Z, Zhou C, Li J, Ye D, Deng H, Cao B, Hao W, Lin L, Zhang Y. SHISA3 Promoter Methylation Is a Potential Diagnostic and Prognostic Biomarker for Laryngeal Squamous Cell Carcinoma. Biomed Res Int 2017;9058749. doi: 10.1155/2017/9058749

21. Chen X, Hu H, Liu J, Yang Y, Liu G Ying X1, Chen Y, Li B, Ye C, Wu $D$, Duan S. FOXF2 promoter methylation is associated with prognosis in esophageal squamous cell carcinoma. Tumour Bio 2017;39(2):1010428317692230.

22. Mzik M, Chmelarova M, John S, Laco J, Slaby, O Kiss I, Bohovicová L, Palicka V, Nekvindova J. Aberrant methylation of tumour suppressor genes WT1, GATA5 and PAX5 in hepatocellular carcinoma. Clin Chem Lab Med 2016;54(12):1971-80.

23. Zhang X, Song YF, Lu HN, Wang DP, Zhang XS Huang SL, Sun BL, Huang ZG. Combined detection of plasma GATA5 and SFRP2 methylation is a valid noninvasive biomarker for colorectal cancer and adenomas. World J Gastroenterol 2015;21(9):2629-37.

24. Rankeillor KL, Cairns DA, Loughrey C, Short SC, Chumas P, Ismail A, Chakrabarty A, Lawler SE, Roberts P. Methylation-specific multiplex ligation-dependent probe amplification identifies promoter meth- ylation events associated with survival in glioblastoma. J Neurooncol 2014;117(2):243-51.

25. Chmelarova M, Krepinska E, Spacek J, Laco J, Nekvindova J, Palicka V. Methylation analysis of tumor suppressor genes in ovarian cancer using MS-MLPA. Folia Biol (Praha) 2012;58(6):246-50.

26. Huang C, Zhou X, Li Z, Liu H, He Y, Ye G, Huang K. Downregulation of thrombospondin-1 by DNA hypermethylation is associated with tumor progression in laryngeal squamous cell carcinoma. Mol Med Rep 2016;14(3):2489-96.

27. Shitani M, Sasaki S, Akutsu N, Takagi H, Suzuki H, Nojima M, Yamamoto H, Tokino T, Hirata K, Imai K, Toyota M, Shinomura Y. Genome-wide analysis of DNA methylation identifies novel cancer-related genes in hepatocellular carcinoma. Tumour Biol 2012;33(5):1307-17.

28. Pesek M, Kopeckova M, Benesova L, Meszarosova A, Mukensnabl P, Bruha F, Minarik M. Clinical significance of hypermethylation status in NSCLC: evaluation of a 30-gene panel in patients with advanced disease. Anticancer Res 2011;31(12):4647-52.

29. Ding N, Bonham EM, Hannon BE, Amick TR, Baylin SB, O'Hagan HM. Mismatch repair proteins recruit DNA methyltransferase 1 to sites of oxidative DNA damage. J Mol Cell Biol 2016;8(3):244-54.

30. Wang YJ, He L, Yuan M, Tsang WW, Hao L, Wang M, Chow LW, Cheung MN, Liu Q, Ng EL, Loo WT, Chow CY, Bai LJ, Yang Z. Epigenetic changes of TIMP-3, GSTP-1 and 14-3-3 sigma genes as indication of status of chronic inflammation and cancer. Int J Biol Markers 2014;29(3),e208-14. 